\title{
Abkürzungen in den Mitteilungen zur Überlieferung
}

\begin{tabular}{|c|c|}
\hline 7br., Sept, Sept., Septbr, & September \\
\hline 9 br, Nov, Nov. & November \\
\hline Apr. & April \\
\hline Aug, Aug. & August \\
\hline$b, b$ & beantwortet \\
\hline beschr. & beschrieben \\
\hline betr., betrl, betrl. & betreffend \\
\hline Bl. & Blatt \\
\hline$D$ & Druck \\
\hline$d, d ., d \ell, d \ell ., \mathrm{d} \ell$ & den \\
\hline Dbr, Dbr., Dec., Xbr, Xbr. & Dezember \\
\hline$E$ & Erstdruck \\
\hline egh. & eigenhändig \\
\hline e, e., erh. & erhalten \\
\hline Erzbischöffl & Erzbischöflich(e) \\
\hline$f$. & folgende \\
\hline Febr. & Februar \\
\hline fr, fr. & frankiert, franco \\
\hline Freyh. & Freiherr \\
\hline Frkfrth & Frankfurt a. $M$. \\
\hline 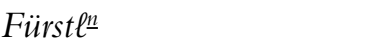 & Fürstlich(en) \\
\hline Geh., Geh: & Geheim(e/er/es) \\
\hline GehRath, Geh. R., & \\
\hline$G . R$ & Geheimrat \\
\hline Geh. Assr & Geheimer Assistenzrat \\
\hline geschriebl & geschrieben \\
\hline$h$ & Abschrift \\
\hline 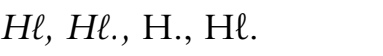 & $\operatorname{Herr}(n)$ \\
\hline$H, H s$ & Handschrift \\
\hline$H d$ & Hand \\
\hline Hochwohlge, & \\
\hline Hochwohlgebl, & \\
\hline Hochwohlgebohrl. & $\begin{array}{l}\text { Hochwohlgeboren } \\
\text { herausoeoehen }\end{array}$ \\
\hline
\end{tabular}


ing $\ell$.

Jan, Jan., Jan.

$J g$

Ju८., Jul.

Jun.

K

Leg. R.

NB.

$\mathrm{Nr}$

Oct, Oct., Octbr

o. $S$.

Prof.

ps., Praes.

Rs., Rücks.

$S$.

Sächs., Sächsl.

Schreiberhd

Sign.

$\mathrm{Slg}$

Sp.

sS

$u, u$.

v., $\mathrm{v}, \mathrm{v}$.

vgl.

Vicepr.

Vol, Vol.

$V s$.

W.

Wohlgl, Wohlgeb.,

Wohlgebe, Wohlgebl.

$z S$

$\ell, \ell$. ingleichen

Januar

Jahrgang

Juli

Juni

Konzept

Legationsrat

lat. nota bene: Wohlgemerkt! Beachte!

Nummer

Oktober

ohne Seite

Professor

lat. präsentatum: eingereicht, überreicht (Tag der

Übergabe)

Rückseite

Seite

Sächsisch(e/en/er/es)

Schreiberhand

Signatur

Sammlung

Spalte

späterer Schreiber

und

von

vergleiche

Vizepräsident

Volumen (lat.: Buch, Band)

Vorderseite

Weimar

Wohlgeboren

zeitgenössischer Schreiber

Abbrechungszeichen 